\title{
Intensité lumineuse et photométrie
}

\author{
M. Pouey
}

Laboratoire de Physique des Gaz et des Plasmas, Bat. 212, Université Paris XI, 91405 Orsay Cedex, France

Résumé: Cet article rappelle les concepts fondamentaux d'intensité lumineuse et les lois générales régissant la propagation des ondes électromagnétiques dans des milieux homogènes. Dans une seconde partie, le vocabulaire de la commission Internationale de l'Eclairage est employé pour décrire les performances des sources de rayonnement dans l'ultraviolet lointain.

Abstract: This paper deals first with the basic concept of luminous intensity and the laws of propagation of the electromagnetic waves. C.I.E. terminology is used, in the second part, to describe the performances of vacuum ultraviolet sources.

\section{LES FONDEMENTS THEORIQUES}

\section{1 Les solutions harmoniques des équations de Maxwell}

Pour un milieu de constante diélectrique $\epsilon$, d'indice de réfraction $n^{2}=\epsilon$ l'intensité de la lumière', définie comme le flux d'énergie du champ électromagnétique, est évaluée par l'amplitude de la moyenne temporelle du vecteur de poynting dont la direction définie la direction de propagation de l'énergie; ces ondes électromagnétiques se propagent à la vitesse $\mathrm{v}=\mathrm{c} / \mathrm{n}$ ( $\mathrm{c}$ étant la vitesse de la lumière dans le vide) [Equations de Maxwell]). Pour des ondes harmoniques monochromatiques de pulsation $\omega$, les champs électriques $\mathbf{E}$ et magnétique $\mathbf{H}$, en un point $P$ de l'espace ( caractérisé par le vecteur $r$ ), sont représentés par des fonctions ( solutions réelles de l'équation d'onde) du type:

$$
\begin{gathered}
V(\mathbf{r}, t)=A(\mathbf{r}) \exp [(i \omega t+\varphi(r)] ; \\
\omega=2 \pi v=\frac{2 \pi}{\mathrm{T}}=\mathrm{v} \frac{2 \pi}{\lambda}=\mathrm{kv}_{i}
\end{gathered}
$$

$v$ étant la fréquence représentant le nombre de vibrations par seconde, $\mathrm{T}$ la période en secondes, $v$ la vitesse en mètres/sec, $\lambda$ la longeur d'onde en nanomètres et $k$ le vecteur d'onde c'est à dire le vecteur de propagation dans le milieu. Les surfaces $\varphi(\mathbf{r})=$ cte sont dénommées surfaces équiphases ou surfaces d'ondes; dans le cas général (ondes inhomogènes) elles ne 
sont pas confondues avec les surfaces d'égale amplitude ce qui est toujours le cas des ondes planes pour lesquelles l'amplitude A est constante [relations d'Helmoltz].

pour ondes de fréquences élevées (qq $10^{14}$ Hertz), les champs électrique et magnétique sont en phase, orthogonaux

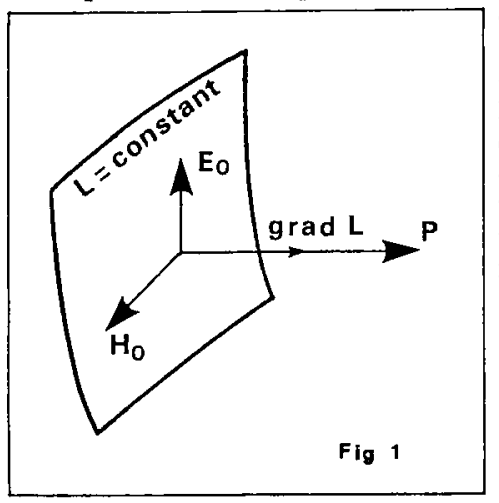
entre eux et tangents à la surface équiphase; la phase de la vibration est égale à $k L(r)$, la fonction $L(\mathbf{r})$, caractérisant la trajectoire des rayons lumineux ( le chemin optique), satisfaisant à la condition grad $\mathbf{L}=\mathrm{n}$ : autrement dit les trajectoires des rayons lumineux sont normales aux surfaces équiphases. Il s'en suit [ principe de Fermat] que le chemin effectivement suivi par la lumiére est une extrêmale du chemin optique $\mathrm{L}$ : dans un milieu uniforme les rayons lumineux se propagent en ligne droite ( Loi de Descartes ; $i=$ $i^{\prime} ; n \sin i=n^{\prime}$ sini'). Dans le cas des réseaux de diffraction ( $I$ défini en coordonnées polaires $w, \ell, \alpha)$, la solution des relations $\alpha^{\prime}$ Helmoltz pour des champs incidents $\mathbf{E}$ ou $\mathbf{H}$ paralléles à la direction $z$ s'exprime par:

$$
v_{z}^{i}=A \exp [i \omega t-i k(w \sin \alpha+\ell \cos \alpha)] \not E_{z}^{i} \text { ou } H_{z}^{i} ;
$$

et pour la totalité des champs diffractés par:

$$
V_{z}^{d}=\int_{-\infty}^{\infty} V_{z}^{d}(\beta) \exp [i k(w \sin \beta+\ell \cos \beta)] d \beta,
$$

$V_{z}^{d}(\beta)$ étant l'amplitude d'une onde "plane diffractée" dans la direction $\beta$. Mais la nature périodique (période d) de la distribution des traits du réseau, sur la totalité de sa surface, implique que le champ électromagnétique total, sur la sưrface du réseau, soit le même pour un déplacement d'un nombre entier de période dans la direction $y ;$ en conséquence:

$$
\begin{gathered}
\mathrm{V}_{z}(\mathrm{w}+\mathrm{pd})=\mathrm{V}_{z}(\mathrm{w}), \\
\left(\mathrm{V}_{z}^{i}+\mathrm{V}_{z}^{d}\right)_{w+p d}=\left(\mathrm{V}_{z}^{i}+\mathrm{V}_{z}^{d}\right)_{w} \quad(\mathrm{p}=\text { entier }) .
\end{gathered}
$$

c'est la périodicité des traits ( $d=1 / \mathrm{N}$ ) qui est à l'origine de l'existence d'ondes diffractêes discrètes :

$$
\sin \alpha+\sin \beta=m \mathbf{N} \lambda,
$$

$\alpha$ étant l'angle d'incidence et $\beta$ l'angle de diffraction, les surfaces équiphases diffractées étant, pour des surfaces d'onde incidentes planes et des réseaux plans, des surfaces d'onde planes.

Si la courbure de la surface d'onde ou celles du composant est grande vis à vis de la longeur d'onde CES PROPRIETES SONT CONSERVEES LOCALEMENT mais le déphasage est augmenté d'une quantité $k$ DL, caractérisant l'écart entre la surface d'onde sphérique et la surface d'onde plane ${ }^{2}$. 
La densité d'énergie de l'onde est égale à $\frac{\epsilon}{4 \pi}$ et, par suite de sa propagation, elie remplit $v \mathrm{~cm}^{3}$ de plus par seconde le flux d'énergie étant défini par le vecteur de poynting $p ; 1 a$ valeur absolue de la moyenne temporelle de ce vecteur caractérise l'intensité lumineuse telle que:

$$
\mathbf{P}=\frac{\mathrm{C}}{4 \pi} \mathbf{E} \wedge \mathrm{H} ; \mathrm{I}=\frac{\mathrm{C} \mathrm{n}}{8 \pi} \mathrm{A}^{2}
$$

L'intensité lumineuse, seule quantité physiquement observable, est donc définie comme la valeur moyenne temporelle de la densité d'énergie qui, par unité de temps, traverse une surface unitaire perpendiculaire à la direction de l'énergie; elle a donc pour dimension $\mathrm{M} \mathrm{T}^{-3}$.

L'onde lumineuse est donc assimilée à une perturbation périodique qui se propage de façon analogue aux vagues à la surface de l'eau. Le vecteur lumineux transversal se comporte, en un point d'un rayon lumineux, comme un bouchon à la surface de l'eau; le long d'un rayon lumineux, à l'instant $t$, la vibration lumineuse est représentée par une sinusoïde dont l'ordonnée est l'extrémité du vecteur lumineux à chaque instant $t$. Bien que le vecteur lumineux, confondu avec la direction du vecteur de Poynting, semble introduire un plan particulier, le rayon lumineux présente les mêmes propriétés dans tous les azimuths: en effet, même pendant un intervalle de temps extrêmement court, l'émission lumineuse est relancée un très grand nombre de fois dans toutes les directions, de façon généralement désordonnée, de telle sorte que le vecteur lumineux voit son orientation et sa phase varier au hasard dans tous les azimuths.

Mais dans les échanges énergêtiques entre la matiére et la lumiére tout se passe comme si la lumière était formēe de particules, les "photons" d'énergie hu (Einstein ). Le principe de "localisation" équivalent au principe d'interférences implique que l'intensité de l'onde mesure la probabilité pour que le photon produise en un point de l'espace un effet observable ( De Broglie $e^{3}$ ).

\section{I.2 Lois de conservation de l'énergie}

Ia loi de conservation de l'énergie implique que la divergence de la valeur moyenne temporelle $\langle\mathbf{p}\rangle$ du vecteur de Poynting soit nulle. Il s'en suit que, pour des ondes planes et des surfaces d'onde sphériques de rayon de courbure $R$, l'intensité lumineuse en chaque point d'un rayon lumineux rectiligne est inversement proportionnelle au carré de $R$. On suppose alors, implicitement, que l'énergie lumineuse tend vers l'infini lorsque la dimension de la source tend vers zéro. De même, si l'on considére un pinceau lumineux issu d'un élément de surface ds sous un angle $\theta$ et dans un angle solide d , l'étendue géométrique $u$ correspondante se conserve (relation de clausius; $n$ et $n^{\prime}$ indices de réfraction ):

$$
\mathrm{U}=\mathrm{n}^{2} \mathrm{ds} \cos \theta \mathrm{d} \Omega=\mathrm{n}^{\prime}{ }^{2} \mathrm{ds} \mathrm{s}^{\prime} \cos \theta^{\prime} \mathrm{d} \Omega^{\prime},
$$


cette relation ne supposant pas, a priori, de correspondance bi-univoque entre un point de la surface ds et de ds' ( elle est à la base de la conception des concentrateurs de lumière).

\section{3 Cas des ondes planes spatialement limitées}

Si, au cours de sa propagation, l'onde plane est limitée spatialement par un contour dont les dimensions sont grandes vis à vis de la longeur d'onde, mais correspondant à de faible valeurs de $d \Omega$, l'intensité lumineuse et le flux d'énergie, pour des champs quasi - monochromatiques non polarisés, peuvent être obtenus à partir d'une fonction d'onde scalaire harmonique complexe ( les composantes cartésiennes des vecteurs $\mathbf{E}, \mathbf{H}$ et $\mathbf{P}$ satisfont cette fonction \}. Après la traversée du contour la distibution spatiale de l'intensité lumineuse n'est plus constante mais présente des" "franges " brillantes et sombres caractéristiques du phénomène de" diffraction ". La formulation mathématique $d u$ principe d'Huygens-Fresnel par Kirchhoff permet alors de définir l'intensité de la lumière comme le carré ( complexe conjugué) de l'amplitude du mouvement vibratoire en tout point $P$ d'une surface d'onde quasi sphérique:

$$
A_{p}=\frac{i}{R \lambda} \iint s^{A_{0}} \exp (i K L) d s
$$

Elle s'obtient en faisant la somme des amplitudes $A_{0}$ distribuées sur la surface d'onde en tenant compte des variations de phase; la décroissance en $1 / R$ de l'amplitude des ondes diffractées traduit la constance de l'énergie au cours de la propagation de l'onde; le facteur $i$ permet de rendre compte du changement de phase au passage du foyer ( la phase de l'onde varie de façon continue, mais elle oscille autour de 0 en avant $\mathrm{du}$ foyer et autour de $\Pi$ aprés); enfin, le facteur $K$ a pour valeur $2 \pi / \lambda$. La quantité $E_{p}=A_{p}$. $A_{p}^{*}$ caractérise alors la répartition spatiale de l'intensité lumineuse au voisinage de tout point $P$ situé sur la surface d'onde.

Les phénomènes de diffraction de Fresnel ( bord d'écran, fil..) se produisent au voisinage de la limite de l'onde et sont éloignés de son centre de courbure. Les phénomènes de diffraction de Fraunhofer sont au contraire localisés au voisinage du centre de courbure de l'onde. La fonction $L$, qui définit, dans le milieu considéré, le temps mis par la lumière pour parcourir la distance qui sépare le point source du point $\mathrm{P}$, est aussi appelée Fonction Caractéristique ou Eikonale ( du

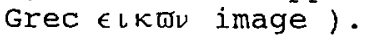

En dénommant respectivement $2 \gamma^{\prime}$ et $2 \beta^{\prime}$ la largeur et la hauteur apparente ( valeurs réelles divisées par la distance image $R$ ) des traits d'un réseau, $\eta=1 / \mathrm{N} R$ le pas apparent du réseau la distribution de l'intensité lumineuse dans un plan perpendiculaire à l'axe optique s'exprime par:

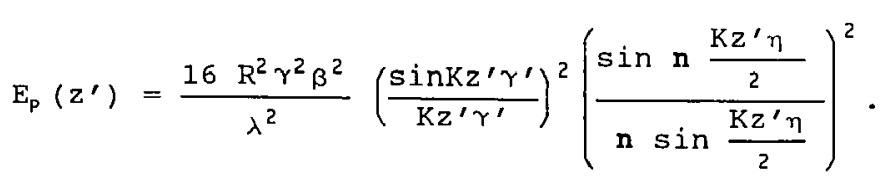


Cette distribution représente la modulation (deuxiéme terme) de la tache de diffraction donnée par une ouverture rectangulaire ( la largeur du trait du réseau ); comme cette dernière dépend, en fait, de la forme des $\mathbf{n}$ silions et qu'elle est spatiallement d'autant plus étalée que $\mathbf{N}$ est grand elle peut être considérée comme constante, la distribution des intensités lumineuse étant alors égale au produit du terme de modulation par $E_{0} / \mathrm{n}^{2}$ si $\mathrm{E}_{0}$ représente l'intensité lumineuse maximale associée aux maxima principaux définis $\operatorname{par} \eta=p \mathbf{N} \lambda$. Au voisinage d'un maximum diffracté dans la direction $\theta$ on peut écrire que $z^{\prime} / R=\sin \left(\theta-\theta^{\prime}\right)=\sin \theta-\theta^{\prime} \cos \theta$, la distribution de l'intensité lumineuse:

$$
E_{p}\left(z^{\prime}\right)=E_{0}\left(\frac{\sin n \frac{K \cos \theta}{2 N} \theta^{\prime}}{n \frac{K \cos \theta}{2 N} \theta^{\prime}}\right) ;
$$

est identique à celle donnée par une fente de largeur $n \cos \theta / N$ c.a.d. de la largeur du faisceau lumineux dans la direction d'observation. Pour un système optique, d'étendue géomètrique limitée, les répartitions spatiales de l'intensité lumineuse $G$,

qui découlent de l'intégrale de kichhoff permettent de caractériser la fonction de transfert de l'instrument qui correspond à la valeur limite théorique, JAMAIS ATTEINTE EN RRATIQUE, de la résolution spatiale de l'instrument. Suivant le critère de Rayleigh, on considére que deux radiations sont séparées lorsque le maximum de l'une des figures de diffraction correspond au premier minimum nul de l'autre, l'écart angulaire correspondant:

$$
\mathrm{d} \beta=\frac{\lambda \mathrm{N}}{\mathrm{n} \cos \beta}=\frac{\lambda}{\mathrm{W} \cos \beta},
$$

définit le fonction de transfert angulaire d'un réseau de largeur $\mathrm{W}$.

Par ailleurs, les phénomènes de diffraction ont été présentés comme une illustration du principe d'incertitude (Eisenberg); la localisation spatiale du rayon lumineux étant d'autant plus déterminée que le contour est de dimension faible, la distribution spatiale de l'intensité lumineuse, après le diaphragme, ne peut ètre définie avec une grande précision. En fait, l'électrodynamique quantique permet d'expliquer l'ensemble de ces phénomènes sans faire appel à ce principe; il suffit de caractériser chacun des points $P_{i}$ d'un rayon lumineux par un vecteur dont l'amplitude est égale à la racine carrée de l'intensité en ce point ( de la probabilité pour que le photon produise en ce point un effet observable, et dont l'orientation est proportionelle au temps mis par la lumière pour aller du point source aux différents points $P_{i} ;$ la somme vectorielle de ces différents vecteurs a pour résultante un vecteur $s_{y}$ dont le carré de l'amplitude défini la probabilité d'un effet observable. Dans le cas de la diffraction cette probabilité n'est pas nulle en dehors de la direction de propagation rectiligne des rayons lumineux. 


\section{TABEAU I DIMENSIONS ET UNITES DES GRANDEURS UTILISEES DANS LE SYSTEME INTERNATIONAL}

$\mathrm{L}$ : longeur ( mètre-m); M: masse (kilogramme-kg);

$\mathrm{T}$ : temps ( seconde-s ); $t$ : température (degré Kelvin- ${ }^{\circ} \mathrm{K}$ )

c: vitesse de propagation de la lumière dans le vide $310^{8} \mathrm{~m} / \mathrm{s}$

$\mathrm{h}$ : constante de Planck $6.6260^{-34}$ Joule seconde

\section{Cas d'une source monochromatique non pulsée \\ GRANDEURS \\ UNITES}

Longeur d'onde $\lambda$ $\lambda 10 \mathrm{eV}$

Nombre d'onde $10 \mathrm{eV}$

Période $10 \mathrm{eV}$

Température $10 \mathrm{eV}$

Vitesse $\mathrm{v}$

Fréquence $\mathbf{f}$

Pulsation $\omega$

$f, \omega 10 \mathrm{eV}$

Charge q

Potentiel V

Polarisation

Champ électrique

Energie U

Densité d'énergie

Energie $1 \mathrm{eV}$

Energie $1 \mathrm{~cm}^{-1}$

Energie 1 Rydberg

Energie $1^{\circ}$ Kelvin

Energie 1 calorie

Puissance $P$

Flux energétique

Flux photonique

Densité de Puissance M / L T $T^{3}$

$\begin{array}{lll}\mathrm{L} / & \mathrm{T} \\ \mathrm{L} & / & \mathrm{T} \\ & \end{array}$

$M L^{2} / T^{3}$ nanomètre (nm)

$123.99 \mathrm{~nm}$;

$1, \lambda=8,065310^{4} \mathrm{~cm}^{-1}$

$\lambda / \mathrm{C}=4,135810^{-6} \mathrm{~s}$

$1,160510^{-4}{ }^{\circ} \mathrm{K}$

$\mathrm{m} / \mathrm{s}$

c / $\lambda$ hertz $\mathrm{Hz}$

$2 \pi \mathrm{f}\left(\mathrm{s}^{-1}\right)$

$2,41810^{15} \mathrm{~Hz}, 1,51910^{16} \mathrm{~s}^{-1}$

q coulomb

$\mathrm{ML}^{2} / \mathrm{g} \mathrm{T}^{2} \quad$ volt

$\mathrm{q}_{\mathrm{M}} / \mathrm{L}^{\mathrm{L}^{\frac{\mathrm{C}^{2}}{}}}$ coulomb $/ \mathrm{m}^{2}$

volt / m

joule ( $\left.{ }^{10} 0^{7} \mathrm{erg}\right)$

joule / $\mathrm{m}^{3}$

$1,60207 \quad 10^{-19}$ joule

$1,986510^{-23}$ joule

$13,606 \mathrm{eV}$

$8,617310^{-5} \mathrm{eV}$

4,185 joule

watt (joule / s)

watt

$5,0310^{15} \mathrm{P} \lambda$ photon / $\mathbf{s}$ $5,0310^{15} \lambda$ photon/joule watt $/ \mathrm{m}^{3}$ 
A partir du moment où l'on veut observer expérimentalement ces répartitions d'intensité lumineuse il est nécessaire de donner au point source une dimension physique (fente de largeur $s$ et de hauteur $h$ dans le cas des réseaux); la résolution angulaire mesurable sera donc toujours supérieure à celle associée a la fonction de transfert. La répartition d'intensité lumineuse dans l'image doit alors être dénommée distribution des éclairements par référence à la photomètrie. II PHOTOMETRIE

La photomètrie ${ }^{4}$ a pour objet la caractérisation de l'énergie lumineuse émise (reçue) par une portion de surface $s$ réelle ou fictive. Cette caractérisation pouvant s'effectuer à l'aide de différents détecteurs, les dimensions et les définitions des différentes grandeurs bien qu'identiques doivent être accompagnées d'épithètes car des unités différentes sont utilisées pour les grandeurs énergétiques et visuelles. Le passage d'un système à l'autre implique notamment la connaissance des de la courbe de visibilité de l'oeil $V_{\lambda}$ (Tableau I; $\mathrm{K}_{\mathrm{m}}=655$ lumen/watt). Par ailleurs, les mesures photomètriques sont toujours effectuèes dans des conditions expérimentales telles que l'approximation de l'optique géometrique soit satisfaite: les dimensions des contours considérés sont donc toujours de dimensions telles que les phénomènes de diffraction puissent être négligés.

\section{II.I Cas d’un milieu non diffusant et d'une lumière mono- chromatique}

A une énergie radiante $U$ (joule) est associé un flux "Flux". $\mid \begin{aligned} & \text { radiant [ flux énergétique, puis- } \\ & \text { sance * d dP a dU/dt (joule/s ou } \\ & \text { watt). Afin d'en étudier les varia- } \\ & \text { tions locales on caractérise le fais- } \\ & \text { ceau lumineux par son étendue gémé- } \\ & \text { trique dG ca.d. par le produit de la } \\ & \text { surface apparente ds cosv par l'angle } \\ & \text { solide dS, le flux radiant étant par } \\ & \text { définition proportionnel à dG: }\end{aligned}$

$$
\mathrm{dP}=\mathscr{R} \mathrm{dG}=\mathscr{R} \mathrm{ds} \cos v \mathrm{~d} \Omega
$$

La quantité $R$, qui représente la densitê spatiale du rayonnement émis par un élément de source ds dans l'angle solide d , est la luminance dans le pinceau lumineux. Elle s'exprime en watt $\mathrm{m}^{-2} \mathrm{sr}^{-1}$ dans le système énergétique International; si le flux est exprimé en nombre de photons/sec la luminance, $s^{\prime}$ exprime en photons Fig 2 Etendue géométrique $\mathrm{s}^{-1} \mathrm{~cm}^{-2} \mathrm{~s} \mathrm{r}^{-1}$. La correspondance entre $d G=d s \cos \nu d \Omega$ les deux systèmes d'unités est associèe au produit de la constante de Planck h ( $\mathrm{h}=6,6240^{-34} \mathrm{Joule-seconde)}$ par la vitesse de la lumière ( $\left.\mathrm{hc}=1.98510^{-25} \mathrm{Joule-m}\right)$ dont la valeur est égale au produit de l'énergie du photons par la valeur de la longeur d'onde. Les autres équivalences du flux radiant, ainsi que les dimensions associées, sont rassemblées dans les tableaux II ( III) pour les sources monochromatiques émettant de façon continue (pulsée). Dans un milieu homogène l'étendue 
géomètrique se conservant, aucun dispositif optique n'est susceptible d'accroitre la luminance d'une source qui ne peut que se dégrader (pertes par réflection ou transmission).

En outre, la luminance d'une source n'est pas toujours uniforme dans l'espace (limitation physique ou mécanique), il est alors nécessaire de définir des indicatrices de luminance.

La quantité dP/ds représente soit l'excitance soit l'éclairement suivant que l'on considére une source de lumiére ou une surface réémettant dela lumiére ( surface diffusante, rayonnement d'un plasma créé par impact laser..) soit l'énergie reçue par une surface (un détecteur). De dimension $M / \mathrm{T}^{3}$ la quantité dp/ds ( $\mathrm{W} \mathrm{m}^{-2}$ ) caractérise donc le flux émis, reçu, par unité d'aire à la surface de la source $s$ ou du récepteur $s^{\prime}$ :

$$
\begin{array}{r}
\varepsilon=\frac{d P}{d s}=\int_{1 / 2} \text { espace } R \cos v d \Omega ; \\
\varepsilon^{\prime}=\frac{d P}{d s^{\prime}}=\int_{1 / 2} \text { espace }{ }^{\Re \cos v \prime} d \Omega^{\prime}
\end{array}
$$

Si la source est orthotrope ( luminance indépendante de l'angle d'observation $v$, l'excitance a pour valeur:

$$
\varepsilon=R \int_{0}^{\pi / 2} 2 \pi \sin \theta \cos \theta d \theta=\pi R ;
$$

il en sera de même, pour un diffuseur orthotrope (diffuseur satisfaisant à l'hypothèse de Lambert ). Si l'élément de surface est orthogonal au vecteur de Poynting, l'intensité lumineuneuse définie au paragraphe précédent a les mêmes dimensions qu'un éclairement !! mais ne peut être quantifiée par des unités puisque le "point source" correspondant est une fonction de Dirac. La répartition des éclairements caractèrise la fonction de transfert (leur résolution limite) des instruments les dimensions du "point source" étant définies par la largeur à mi-hauteur de la tache de diffraction lièe à la pupille d'entrée.

L'intensité d'une source dans une direction donnée est, en photometrie, la valeur du rapport entre le flux émis par l'ensemble de la source dans une direction donnée et dans l'angle solide dans lequel le flux est recueilli. L'intensité lumineuse s'exprime alors en candéla qui est l'une des sept unités fondamentales du système International. Au niveau expérimental, le diamètre apparent du "point source" ne doit pas être inférieur au vingtième de la distance d'observation.

La quantité de lumière (joule, nombre de photons, lumenseconde, est l'intégrale du flux transporté par un faisceau pendant un intervalle de temps donné; de même l'exposition est l'intégrale de l'éclairement d'une surface réceptrice pendant un intervalle de temps donné $\left(\mathrm{J} / \mathrm{m}^{2}, \mathrm{~m}^{-2}\right.$, lux.s $)$. 
II GRANDEURS RADIOMETRIQUES [R] ET PHOTOMETRIQUES [P]

Etendue géomètrique: $\mathbf{d g}=\mathrm{ds} \cos \theta \mathrm{d} \Omega$

\section{Grandeurs}

[R] Energie radiante

Dêfinitions

v

$\mathbf{Q}=\mathbf{R}_{\mathrm{m}} \mathbf{v}_{\lambda}$

$P=d u / d t$

$F=d Q / d t$

[P] Flux lumineux

[R] Radiance

[P] Luminance

$\Re=\mathbf{d P} / \mathbf{d G}$

$\mathfrak{L}=\mathbf{d F} / \mathbf{d G}$

Unités

[R] Eclairement énergétique $\mathrm{E}$
Exitance energ.

$\varepsilon=\mathbf{d p} / \mathbf{d s}$

W $\mathrm{m}^{-2}$

[P] Eclairement lumineux

Exitance lumineuse

$\varepsilon \stackrel{E}{=} \mathbf{d} F / \mathbf{d s}$

$\operatorname{Lux}=\mathrm{Lm} \mathrm{m}^{-2}$

[R] Intensité énergétique $I=\mathbf{d} \boldsymbol{F} / \mathbf{d} \Omega$

$\mathrm{W} \operatorname{sr}^{-1}$

[P] Intensité lumineuse $\quad \mathbf{I}=\mathbf{d} \mathbf{F} / \mathbf{d} \Omega$

Candela

Talbot

Watt $=$ Joule $s^{-1}$

Lumen $=$ Talbot $\mathrm{s}^{-1}$

$\mathrm{W} \mathrm{m}^{-2} \mathrm{sr}^{-1}$

Lm $\mathrm{m}^{-2} \mathrm{sr}^{-1}$

On appelle diffuseur orthotrope un échantillon diffusant qui réfléchirait la lumière reçue en la restituant avec une luminance independante de la direction de renvoi:

Exitance: $R$ E; Luminance $\mathcal{L}=R E / \pi$

Sources émettant une luminance $\mathcal{L}$ uniforme ( Excitance $\pi \mathfrak{L}$ ) Filament de tungstène, salycilate de sodium

Type 1: Plan 1 face d'aire $A$

Intensité: $I=I A \cos \theta$

Indicatrice: sphère tangente au plan

Flux: $F=\pi$ L A

Type 2: sphère de rayon $r A=4 \pi r^{2}$

Intensité: $I=\pi r^{2} \mathrm{I}$

Indicatrice: sphère centrée sur la source

Flux: $F=(2 \pi r)^{2} L$

Type 3: cylindre longeur $\ell$, diamètre $d$, surface latérale $A=\pi d \ell$

Intensité: $I=\mathrm{d} \ell \mathrm{I} \cos \theta$

Indicatrice: tore axé sur le cylindre, cercle

générateur tangent à l'axe

Flux: $F=\pi^{2} \mathrm{~d} \ell \mathrm{L}$ 


\section{TABLEAU III RAYONNEMENT LASER CONTINU ET EN IMPULSION}

Energie (joule); Puissance (watt); section (micron carré)

Densité d'énergie électromagnétique $\left(\mathrm{J} / \mathrm{cm}^{3}\right)$.

Longeur (microns); temps ( $\tau$ en ps); longeur (micron)

\section{GRANDEURS}

$\begin{array}{ll}\text { Pulsation } & \omega=2 \pi c / \lambda \\ \text { Période } & \mathrm{T}=2 \pi / \omega \\ \text { Longeur impulsion } & \ell=c \tau \\ \text { Irradiance } & \mathrm{P} / \mathrm{s} \\ \text { Champ électrique } & 2 \mathrm{I} / \mathrm{c}, \\ \text { Nombre de photons } & \mathrm{I} \tau / \hbar \omega \\ \text { Densité d'ênergie } & \epsilon_{0} \mathrm{E}_{0}^{2} / 2\end{array}$

DENOMINATION/UNITES USUELLES

Le champ subit par un électron sur la première orbite de Bohr est de $5,110^{9} \mathrm{v}, \mathrm{cm}$, et celui ressenti par un nucléon au sein du noyau de $1^{\prime}$ ordre de $10^{18} \mathrm{v}, \mathrm{cm}$. Un corps noir de tempéra ture $T(\mathrm{KeV})$ rayonne une densité d'énergie $3,410^{6} \mathrm{~T}^{4} \mathrm{~J} / \mathrm{cm}^{3}$; les densités d'énergie laser et de corps noir sont égales pour $T=$ $0,17 \quad 10,25 \mathrm{keV}$. Un laser HeNe de $2 \mathrm{~mW}\left(6,410^{15}\right.$ photons $/ \mathrm{s}$ soit $3,210^{18}$ photons/joule) focalisé par une lentille de $5 \mathrm{~mm}$ de focale produit une irradiance continue de $9.7910^{3} \mathrm{H} / \mathrm{cm}^{2}$ ( diamètre de $I^{\prime}$ image $5,1 \mu \mathrm{m}$ ). De même un laser $\mathrm{CO} 2$ de $5 \mathrm{~kW}$ peut être focalisé sur un diamètre de $0,5 \mathrm{~mm}$ (miroirs en silicium polycristallin massif refroidis "sous la peau" pour minimiser les déformations de surface sous haut flux, , l'irradiance produite en continu étant de $2,5510^{4} \mathrm{~W} / \mathrm{cm} \mathrm{m}^{2}$.

Les lasers ( $\mathrm{XeCl}-308 \mathrm{~nm}$ ) en impulsion sont caractérisés par 1 'énergie par impulsion $(0,3 \mathrm{~J})$ et la fréquence de répétition maximale ( $10 \mathrm{~Hz})$ dont le produit défini la puissance moyenne ( $3 \mathrm{~W})$; la notion de puissance moyenne a été introduite dans un souci de classification mais ne: constitue pas une caractéristique fondamentale du laser ( une puissance moyenne de $1 \mathrm{KW}$ peut être obtenue avec un laser délivrant des impusions de 1 joule si la fréquence est de $1 \mathrm{kHz}$ ou de $10 \mathrm{~J}$ si la fréquence est de $100 \mathrm{~Hz}$; le choix de la fréquence va dépendre des applications envisagées ). Il est donc nécessaire de définir, en outre, la durée de l'impulsion ( 40 ns) pour pouvoir évaluer la puissance crête ( puissance délivrée pendant l'impulsion; $7,510^{6} \mathrm{~W}$ ). La dernière caractéristique est la divergence $(0,5 \mathrm{mrad}$ avec une cavité instable ) qui permet de définir la surface minimale $\left(10 \mathrm{~mm}^{2}\right.$ ) obtenue, en pratique, aprés focalisation et d'évaluer l'éclairement $\left(7,510^{15} \mathrm{H} / \mathrm{cm}^{2}\right.$ ) pendant l'impulsion laser (des éclairements de $10^{16} \mathrm{~W} / \mathrm{cm}^{2}$ ont été obtenus à $248 \mathrm{~nm}$ au Laser Facility d'Héraklion ).

Les termes d'usage, "Intensité" pour irradiance pendant l'impulsion laser ou "Fluence" en $\mathrm{J} / \mathrm{cm}^{2}$, sont, dans le cadre des recommandations de la commission Internationale d'Eclairagisme, à prohiber.

[d'après un fascicule de G.Bonnaud pour le DEA Physique des Gaz et des Plasmas et les catalogues d'ORIEL, MELLES GRIOT et SOPRA ]. 
II.2 Cas d'un milieu non diffusant et d'une lumière polychromatique

Dans le cas d'un rayonnement polychromatique continu et calculable théoriquement les unités énérgétiques et photoniques précédentes peuvent être conservées à condition de définir un intervalle spectral large. Par exemple, à $17^{\circ} \mathrm{C}(290 \mathrm{~K})$ un corps noir émet $1,1810^{22}$ photon par seconde, mètre carré et stéradian; $98 \%$ des photons sont émis entre 6,11 et $195 \mu \mathrm{m}$; dans le visible la luminance est de un photon par mètre carré et stéradian toutes les 2000 secondes. Pour une lampe à tungstène ( $2900 \mathrm{~K}) \mathrm{la}$ luminance à $10 \mu \mathrm{m}$ est 10000 fois plus grande; $9,45 \%$ de la puissance est émise dans le visible et $0,15 \%$ en dessous de $400 \mathrm{~nm}$.

En règle générale, la plupart des rayonnements sont composès de photons dont les énergies $u$, donc les longeurs d'onde $\lambda$, sont très diverses; la distribution spectrale, généralement complexe, des grandeurs photomètriques $R$ est alors caractérisée par la fonction "spectrique" associée $R(\lambda)$ qui caractérise la limite, lorsque $\delta \lambda$ tend vers 0 de $\delta R / \delta \lambda, \delta R$ étant la fraction de $R$ contenue dans la bande spectrale de largeur $\delta \lambda$ mesurée autour de la longeur d'onde $\lambda$. Les grandeurs spectriques sont évaluées en unitês photoniques, les longeurs $\mathrm{d}^{\prime}$ onde en $\mu \mathrm{m}$ ou $\mathrm{nm}$. Pour une raie isolée ou un spectre continu, le flux énergétique est relié au flux photonique par la relation:

$$
\text { Flux energétique } P_{e}=u P_{p}=\frac{h c}{\lambda} P_{p} ;
$$

Le flux spectrique énergétique $\mathrm{P}_{\mathrm{e}}^{\prime}$ étant relié au flux spectrique photonique par la relation:

$$
P_{p}^{\prime}(u)=\left(\frac{\lambda^{3}}{\left(h(c)^{2}\right.}\right) P_{e}^{\prime}(\lambda)=\left(\frac{h c}{u^{3}}\right) P_{e}^{\prime}(\lambda) ;
$$

Et dans le cas général on écrira:

$$
P_{p}=s P_{p}^{\prime}(u) d u ; P_{e}=\int u P_{p}^{\prime} d u ;
$$

A titre d'exemples, la luminance spectrique d'un mini arc à $\operatorname{Argon}^{5}$ ( courant de 50 ampère) croit de $510^{-2}$ à $10^{-1}$ watt / $\mathrm{cm}^{2} \mathrm{~nm} \mathbf{s r}$ entre 200 et $300 \mathrm{~nm}$; elle est, à $300 \mathrm{~nm}$, respectivement pour le soleil (au dessus de l'atmosphère), un arc au $x$ énon et une lampe halogène (tungstène-quartz) de $0,75,0,085$ et $0,0015 \mathrm{w} \mathrm{cm}^{-2} \mathrm{~s} \mathrm{r}^{-1} \mathrm{~nm}^{-1}$. Dans le cas du Rayonnement synchrotron, le terme d'usage "brillance spectrale" doit être dénommé Luminance spectrique et, du point de vue de l'utilisateur, être exprimées par stéradian et par nanomètre. Les données ( $\mathrm{fig.3}$ document du CEA de Limeil Brévannes) sont en pratique gênéralement exprimées photons par seconde millimillimètre carré milliradian carré et " une bande passante de $0,1 \%$ "c.a.d. pour un pouvoir de résolutionde 1000. En admettant que la largeur et la hauteur de la fente d'entrée sont accordées à la dimension de la "source" ou de son image par un 


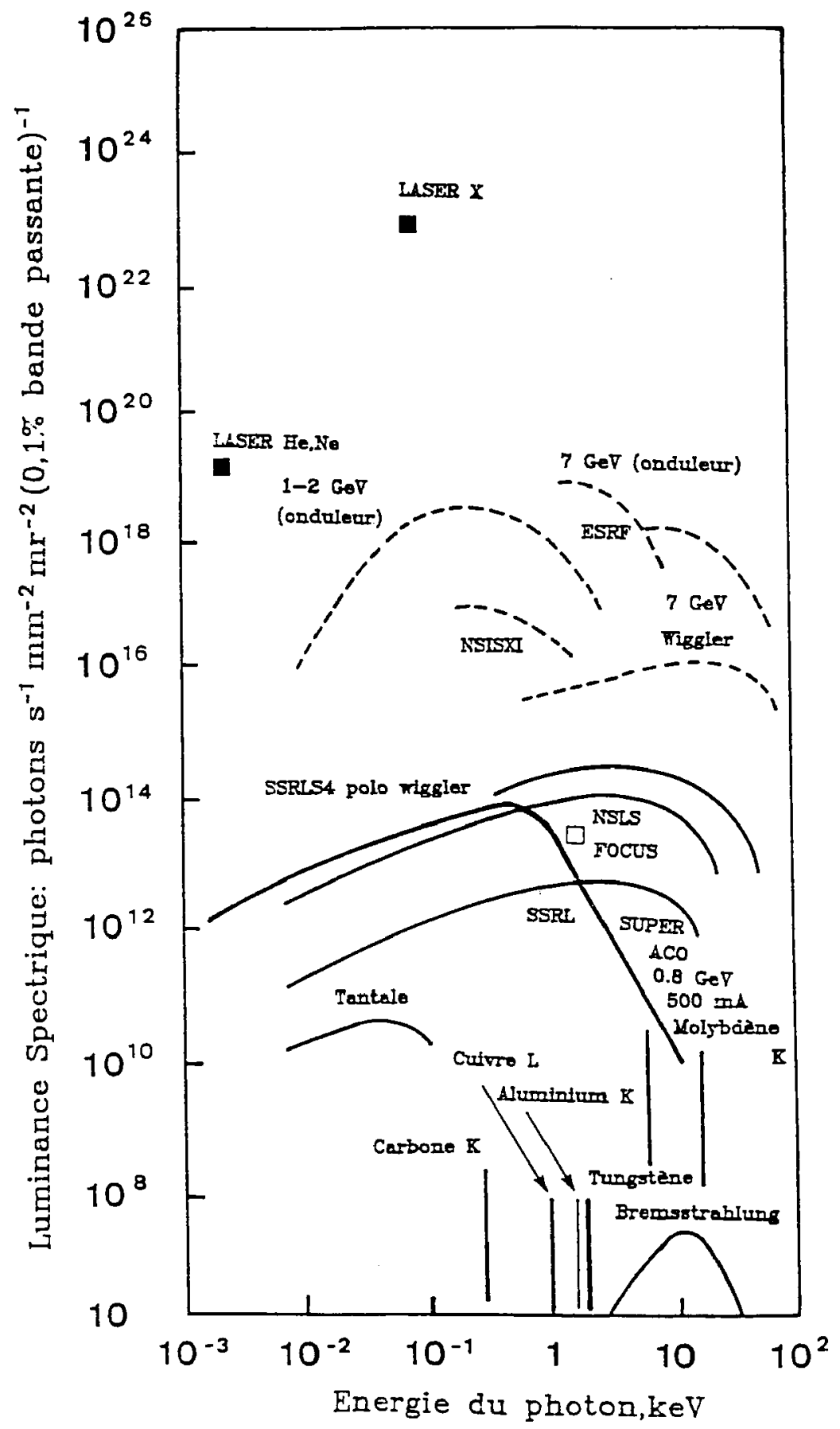


miroir torique, si $\mathbf{k}$ est l'éfficacité globale (pouvoir réflecteur et pertes par astigmatisme) du système optique dispersif le flux disponible à la sortie s'exprimera par:

$$
\mathbf{P}_{\mathrm{p}}=\mathrm{L}_{\mathrm{p}}^{\prime} \lambda\left(\frac{\Delta \lambda_{\mathrm{s}}}{\lambda}\right)^{2} \mathrm{~W} \mathrm{~L} \quad \mathrm{~K} \frac{\mathrm{h}}{\mathrm{r}} \text { photons / seconde. }
$$

A Bessy ${ }^{6}$ des flux $1,510^{8}(500 \mathrm{eV})$ et de $210^{7}(1700$ $\mathrm{eV}$ ) photons par seconde et par $\mathrm{mA}$ de courant ont été mesurés récemment.

\section{CONCLUSION}

Les définitions et dénominations utilisées dans cet article sont celles recommandées par la commission Internationale de l'Eclairage (CIE) et la Commission Electrotechnique Internationale qui $n^{\prime}$ accorde un caractère légal qu'aux unités énergétiques et visuelles mais qui reconnait néanmoins le système photonique. La Norme Française NF C01 845 de mars 1989 stipule l'abandon de termes et d'unités anciennes: par exemple, à la notion d' "éclat" $(<1924)$ a été substitué, jusqu'en 1948, le terme de brillance dont la dénomination officielle est désormais celui de luminance. De même au terme de radiance (<1954) a été substitué, jusqu'en 1970 le terme d'émittance dont la dénomination officielle est l'exitance.

Par ailleurs, dans la littérature anglo-saxone, à l'illuminance évaluée en foot-candle correspond l'éclairement lumineux $(10,764$ lux $)$, à une luminance de 1 foot-lambert correspond une luminance lumineuse de $3,42 \mathrm{~cd} \mathrm{~m}^{-2}$; aux termes d'irradiance et de radiance correspondent l'éclairement énergétique et la luminance énergétique. Les anglo-saxons, en effet, utilisent un vocabulaire différent pour les mêmes grandeurs suivant qu'elles sont exprimées en unités énergétiques (radiometry) ou lumineuses (photometry); cette distinction n'est pas rationnelle dans l'esprit de la langue française, car les grandeurs sont dimensionnellement identiques, seul le système d'unités ( toujours au singulier) change. Ainsi au terme anglo-saxon de fluence employé par la communauté des utilisateurs de Lasers en impulsion correspond celui d'exposition énergétique (joule par mètre carré), photonique (photon par mètre carré) ou lumineuse (lux seconde).

\section{REFERENCES}

1) Born, M. \& Wolf, E. (1975) "Principles in optics" Pergamon Oxford.

2) Maréchal, A. (1957) "Imagerie Géomètrique, Aberrations" Masson, Paris.

3) De Broglie, L.(1956) "Tentative d'Interprétation Causale et Non Linéaire de la Mécanique ondulatoire" GauthiersVillars, Paris.

4) Desvignes, F.(1991) "Rayonnements optiques" Masson, Paris.

5) Croche, R., J. Sci. Instrum., 1990, 13, 165.

6) Krumerey, M., Jahresbericht, 1990, 84 . 